\title{
ON THE SECOND MAIN THEOREM OF CARTAN
}

\author{
Alexandre Eremenko* \\ Purdue University, Department of Mathematics \\ West Lafayette, IN 47907, U.S.A.; eremenko@math.purdue.edu
}

\begin{abstract}
The possibility of reversion of the inequality in the Second Main Theorem of Cartan in the theory of holomorphic curves in projective space is discussed. A new version of this theorem is proved that becomes an asymptotic equality for a class of holomorphic curves defined by solutions of linear differential equations.
\end{abstract}

\section{Introduction}

We consider holomorphic curves $f: \mathbf{C} \rightarrow \mathbf{P}^{n}$. In homogeneous coordinates such curves are represented as $(n+1)$-tuples of entire functions

$$
f=\left(f_{0}: \ldots: f_{n}\right),
$$

where not all $f_{j}$ are equal to 0 . A homogeneous representation is called reduced if the $f_{j}$ do not have zeros common to all of them. A reduced representation is defined up to a common entire factor which is zero-free.

In the following definitions we use a reduced homogeneous representation, however one can easily check that the definitions of $N(r, a, f), T(r, f), N_{1}(r, f), m(r, a, f)$ and $m_{k}(r, f)$ are independent of the choice of a reduced homogeneous representation.

Let $a$ be a hyperplane in $\mathbf{P}^{n}$. It can be described by an equation

$$
\alpha_{0} w_{0}+\ldots+\alpha_{n} w_{n}=0, \quad \text { where } \quad \alpha=\left(\alpha_{0}, \ldots, \alpha_{n}\right) \neq(0, \ldots, 0) .
$$

The intersection points of the curve $f(z)$ with the hyperplane $a$ are zeros of the entire function $g_{a}=(\alpha, f)=\alpha_{0} f_{0}+\ldots+\alpha_{n} f_{n}$. Let $n(r, a, f)$ be the number of these zeros in the disc $|z| \leq r$, counting multiplicity, then the Nevanlinna counting function is defined as

$$
N(r, a, f)=\int_{0}^{r}(n(t, a, f)-n(0, a, f)) \frac{d t}{t}+n(0, a, f) \log r .
$$

The Cartan-Nevanlinna characteristic $T(r, f)$ can be defined as follows:

$$
\begin{aligned}
T(r, f) & =\frac{1}{2 \pi} \int_{0}^{r}\left(\int_{|z| \leq t} \Delta \log \|f(z)\| d m_{z}\right) \frac{d t}{t} \\
& =\frac{1}{2 \pi} \int_{-\pi}^{\pi} \log \left\|f\left(r e^{i \theta}\right)\right\| d \theta-\log \|f(0)\|,
\end{aligned}
$$

where $\|f\|=\sqrt{\left|f_{0}\right|^{2}+\ldots+\left|f_{n}\right|^{2}}$, and $d m$ is the element of the area. Here $\Delta \log \|f\|$ is the density of the pull-back of the Fubini-Study metric, and equality holds by

doi:10.5186/aasfm.2014.3937

2010 Mathematics Subject Classification: Primary 30D35, 32A22.

Key words: Nevanlinna theory, Second Main theorem, linear differential equations.

*Supported by the NSF grant DMS-1361836. 
Jensen's formula. The order $\rho$ of $f$ is defined by the formula

$$
\rho=\limsup _{r \rightarrow \infty} \frac{\log T(r, f)}{\log r} \text {. }
$$

The proximity functions are

$$
m(r, a, f)=\frac{1}{2 \pi} \int_{-\pi}^{\pi} \log \frac{\|\alpha\| \| f\left(r e^{i t} \|\right.}{\left|g_{a}\left(e^{i t}\right)\right|} d t .
$$

Here the integrand is

$$
\log \frac{1}{\operatorname{dist}(f(z), a)}
$$

where dist is the "chordal distance" from the point $f(z)$ to the hyperplane $a$. Now we consider the Wronskian determinant $W_{f}=W\left(f_{0}, \ldots, f_{n}\right)$ which is an entire function; it is identically equal to zero if and only if $f$ is linearly degenerate, that is if $f_{0}, \ldots, f_{n}$ are linearly dependent. We denote by $n_{1}(r, f)$ the number of zeros of $W_{f}$ in the disc $\{z:|z| \leq r\}$ and define the function $N_{1}(r, f)$ by a formula similar to (2).

A set $A$ of hyperplanes is usually called admissible if any $n+1$ hyperplanes of the set have empty intersection. If the set $A$ contains at least $n+1$ hyperplanes, admissibility is equivalent to

$$
\operatorname{codim}\left(a_{1} \cap \ldots \cap a_{k}\right)=k
$$

for every $k \in[1, n+1]$ and every $k$ hyperplanes of the set $A$. We use the convention that $\operatorname{codim} x=n+1$ iff $x=\emptyset$. We use (3) to extend the definition of admissibility to systems of arbitrary cardinality. So a system of hyperplanes will be called admissible if any $k \leq n+1$ vectors $\alpha$ defining these hyperplanes as in (1) are linearly independent.

With these definitions, the Second Main Theorem (SMT) of Cartan says:

For every linearly non-degenerate holomorphic curve and for every finite admissible set $A$,

$$
\sum_{a \in A} m(r, a, f)+N_{1}(r, f) \leq(n+1) T(r, f)+S(r, f)
$$

where $S$ is an "error term" with the property that $S(r, f)=o(T(r, f))$ for $r \rightarrow \infty, r \notin$ $E$, where $E$ is an exceptional set of finite length.

Better estimates of the error term are available, but they do not concern us here. When $n=1$, Cartan's SMT coincides with the Second Main Theorem of Nevanlinna for the meromorphic function $f=f_{1} / f_{0}$. When $n=1$, the assumption that the set $A$ is admissible is vacuous.

Nevanlinna's SMT was considered from the very beginning as a partial generalization of the Riemann-Hurwitz formula [2]. However, the Riemann-Hurwitz formula is an equality, while the SMT is only an inequality. This inspired the research on the reversion of the SMT: roughly speaking, the question is whether one can replace the $\leq$ sign with the $=\operatorname{sign}$ in (4) for $n=1$. A survey of the early results on this topic is contained in the book by Wittich [16, Ch. IV]. The general conclusion one can make from these results is that for all simple, "naturally arising" meromorphic functions an asymptotic equality indeed holds. But of course, (4) cannot be literally true for all meromorphic functions in the form of equality, because there are meromorphic functions $f$ with $m(r, a, f) \neq o(T(r, f))$ for an uncountable set of $a$, and an exceptional set $E$ of $r$ does not help. 
Recently, Yamanoi [17] found a way to overcome this difficulty for $n=1$. He defined the modified proximity function

$$
\bar{m}_{q}(r, f)=\sup _{\left(a_{1}, \ldots, a_{q}\right) \in \overline{\mathbf{C}}^{q}} \frac{1}{2 \pi} \int_{-\pi}^{\pi} \max _{1 \leq j \leq q} \log \frac{1}{\operatorname{dist}\left(f\left(r e^{i t}\right), a_{j}\right)} d t .
$$

With this definition, he proved the following theorem.

Let $f: \mathbf{C} \rightarrow \overline{\mathbf{C}}$ be a transcendental meromorphic function. Let $q: \mathbf{R}_{>0} \rightarrow \mathbf{N}$ be a function satisfying

Then

$$
q(r) \sim\left(\log ^{+} \frac{T(r, f)}{\log r}\right)^{20}
$$

$$
\bar{m}_{q(r)}(r, f)+N_{1}(r, f)=2 T(r, f)+o(T(r, f)), \quad r \notin E,
$$

where $E$ is a set of zero logarithmic density.

For functions of finite order, this result was improved in [18]: it holds with any function $q(r)$ that satisfies $\log q(r)=o(T(r, f))$.

In this paper, we discuss the possibility of an asymptotic equality in Cartan's SMT for arbitrary $n>1$. First we show by an example that the admissibility condition creates a new difficulty which is not present for $n=1$ : even for very simple curves there can be no admissible system for which (4) holds with equality. Then we propose a modified form of Cartan's SMT which does not involve the admissibility condition, and show that in this modified form asymptotic equality holds for a class of holomorphic curves.

\section{Example}

The simplest non-trivial examples in value distribution theory for $n=1$ are meromorphic functions $f=w_{1} / w_{0}$, where $w_{0}, w_{1}$ are two linearly independent solutions of a differential equation of the form

$$
w^{\prime \prime}+P w=0,
$$

where $P$ is a polynomial. These functions $f$, which were studied in detail by F. Nevanlinna [9] and R. Nevanlinna [10], can be characterized by the properties: $f$ is of finite order, and $N_{1}(r, f) \equiv 0$.

For each such $f$, there is an integer $p$ and a finite set of points $\left\{a_{1}, \ldots, a_{q}\right\}$ in $\overline{\mathbf{C}}$ such that

$$
m\left(r, a_{j}, f\right)=\left(2 m_{j} / p\right) T(r, f)+O(\log r), \quad r \rightarrow \infty,
$$

where $m_{j}$ are positive integers, and

$$
\sum_{j=1}^{q} m_{j}=p .
$$

So we have an asymptotic equality in (4).

This result is related to two other results:

1. If $f$ is a meromorphic solution of arbitrary linear differential equation with polynomial coefficients, then we have an asymptotic equality in the SMT for $f$, with $A=\{0, \infty\},[16$, Ch. IV]. 
2. If $f$ has finitely many critical and asymptotic values, then an asymptotic equality holds in the SMT for $f$, if $A$ is the set of critical and asymptotic values $[13,16]$. Functions $f=w_{1} / w_{0}$, where $w_{0}, w_{1}$ are linearly independent solutions of (5) have no critical values and their asymptotic values are exactly those $a_{j}$ in (6).

These results suggest that in searching for improvements of (4) one has to look first at the holomorphic curves whose homogeneous coordinates are linearly independent solutions of a differential equation

$$
w^{(n+1)}+P_{n} w^{(n)}+\ldots+P_{0} w=0,
$$

with polynomial coefficients $P_{j}$. This class of curves can be characterized by the properties that the order is finite and $N_{1}(r, f) \equiv 0,[11,6]$.

The following example was mentioned in [5]:

$$
w^{\prime \prime \prime}-z w^{\prime}-w=0 .
$$

This is equivalent to

$$
w^{\prime \prime}-z w=c, \quad c \in \mathbf{C} .
$$

This is a non-homogeneous Airy equation, and we can describe the asymptotic behavior of all solutions using the well-known asymptotic formulas [1, 14]. All non-trivial solutions are entire functions of order $\rho=3 / 2$, and for description of their behavior we use the Phragmén-Lindelöf indicator:

$$
h_{w}(t)=\lim _{r \rightarrow \infty} r^{-3 / 2} \log \left|w\left(r e^{i t}\right)\right| .
$$

First of all, we have three solutions $w_{0}, w_{1}, w_{2}$ (Airy's functions) for $c=0$. These satisfy

$$
w_{0}+w_{1}+w_{2}=0
$$

and have the indicators

$$
H_{0}(t)=-\cos \left(\frac{3}{2} t\right), \quad|t| \leq \pi, \quad H_{j}(t)=H_{0}(t \pm 2 \pi / 3), \quad j=1,2 .
$$

The rest of solutions of (8), which correspond to non-zero values of $c$ in (9) can be expressed in terms of Airy functions by the method of variation of constants. These explicit asymptotic expressions show that the list of possible indicators for $c \neq 0$ is this:

$$
G_{0}(t)=\left(-\cos \left(\frac{3}{2} t\right)\right)^{+}, \quad|t| \leq \pi, \quad G_{j}(t)=G_{0}(t \pm 2 \pi / 3), \quad j=1,2 .
$$

Another way to obtain these indicators is to notice that (8) has a formal solution

$$
w^{*}(z)=\sum_{n=0}^{\infty} \frac{(3 n) !}{3^{n} n !} z^{-3 n-1} .
$$

According to the general theory [14], there exists a solution $w_{3}$ such that $w_{3}(z)$ has $w^{*}$ as the asymptotic expansion in the sector

$$
S_{0}=\{z:|\arg z|<\pi / 3\} .
$$

For this solution, $h_{w_{3}}(t)=0,|t| \leq \pi / 3$. As the equation (8) is invariant under the substitution $z \mapsto e^{2 \pi i / 3} z$, in each of the three sectors $S_{0}, S_{ \pm 1}=e^{ \pm 2 \pi i / 3} S_{0}$ there exists a solution with zero indicator. 
Notice that for every $t \notin\{\pi, \pm \pi / 3\}$, the set of solutions with $h_{w}(t) \leq 0$ is at most two dimensional. Indeed, if there were three linearly independent solutions with $h_{w}(t) \leq 0$, then every solution would satisfy $h(t) \leq 0$, but this is not so because $\max \left\{H_{0}, H_{1}, H_{2}\right\}$ is positive at every point $t \notin\{\pi, \pm \pi / 3\}$. As for every $t$ there exists a solution $w$ with $h_{w}(t)=0$, we obtain that for every $t$, the set of solutions $w$ with $h_{w}(t)<0$ is at most one-dimensional. This shows that our list (11), (12) of possible indicators of solutions is complete.

Now let $f$ be the holomorphic curve whose homogeneous coordinates are three linearly independent solutions of (8). Then the entire functions $g_{a}=(a, f)$ are exactly the non-trivial solutions of (8). Let $A=\left\{a_{1}, \ldots, a_{q}\right\}$ be an admissible system of hyperplanes. Let $h_{j}$ be the indicators of entire functions $g_{a_{j}}$, and let $h$ be their pointwise maximum. Then

$$
h(t)=|\cos ((3 / 2) t)|, \quad \frac{1}{2 \pi} \int_{-\pi}^{\pi} h(t) d t=\frac{3}{2 \pi} \int_{-\pi / 3}^{\pi / 3} \cos \left(\frac{3}{2} t\right) d t=\frac{2}{\pi},
$$

therefore

$$
T(r, f)=\left(\frac{2}{\pi}+o(1)\right) r^{3 / 2}
$$

We claim that

$$
\sum_{j=1}^{q} \int_{-\pi}^{\pi}\left(h(t)-h_{j}(t) d t \leq 8 \int_{-\pi / 3}^{\pi / 3} \cos \left(\frac{3}{2} t\right)=\frac{32}{3} .\right.
$$

This follows from the fact that on each of the three components of the set $\{t \in$ $(-\pi, \pi): h(t)>0\}$ at most one of the $h_{j}$ can be negative, and at most two of the $h_{j}$ can be non-positive, and in addition, we cannot have negative indicators in all three components, because the three solutions $w_{0}, w_{1}, w_{2}$ satisfying (10) cannot be all present in an admissible set. So we have

$$
\sum_{j=1}^{q} m\left(r, a_{j}, f\right) \leq\left(\frac{16}{3 \pi}+o(1)\right) r^{3 / 2} \leq\left(\frac{8}{3}+o(1)\right) T(r, f) .
$$

As the Wronski determinant of three linearly independent solutions of (8) is zero-free, $N_{1}(r, f) \equiv 0$, and we cannot have asymptotic equality in (4).

This example shows that if one desires (4) with asymptotic equality then nonadmissible sets of hyperplanes $A$ should be permitted. In the next section we state and prove a version of (4) which applies to an arbitrary finite system of hyperplanes.

\section{Modified Second Main Theorem}

Let us consider the projective space $\mathbf{P}^{n}$ equipped with the chordal metric dist. The distance between two subsets of $\mathbf{P}^{n}$ is defined in the usual way, as the $\inf \operatorname{dist}(x, y)$, where $x$ is in one set and $y$ is in another set.

Let us fix an arbitrary finite set $A$ of hyperplanes. Intersections of various subsets of hyperplanes in $A$ are projective subspaces of various codimension. We call these subspaces "flats generated by $A$ ", and denote the set of all these flats by $F(A)$. We also denote by $\operatorname{codim}(x)$ the codimension of a flat $x \in F(A)$. If $\operatorname{codim}(x)=k$, then there exists an admissible set $\left\{a_{1}, \ldots, a_{k}\right\} \subset A$ such that $x=a_{1} \cap \ldots \cap a_{k}$. If $\emptyset \in F(A)$, then flats of all codimensions $1, \ldots, n+1$ exist in $F(A)$. Such systems 
$A$ will be called complete. A system of hyperplanes is complete if the vectors $\alpha$ corresponding to this system as in (1) span $\mathbf{C}^{n+1}$.

We frequently use the following fact, without special mentioning: if $a_{1}, \ldots, a_{k}$ is an admissible set of hyperplanes, and $X=a_{1} \cap \ldots \cap a_{k}$ then

$$
C_{1} \max _{1 \leq j \leq k} \operatorname{dist}\left(w, a_{j}\right) \leq \operatorname{dist}(w, X) \leq C_{2} \max _{1 \leq j \leq k} \operatorname{dist}\left(w, a_{j}\right), \quad w \in \mathbf{P}^{n},
$$

with positive constants $C_{1}, C_{2}$ depending only on the set of hyperplanes.

For $w \in \mathbf{P}^{n}$ and $k \in\{1, \ldots, n\}$, we define $d_{j}(w)$ as the shortest distance from $w$ to a flat of codimension $k$ in $F(A)$. It is also convenient to set $d_{n+1}(w)=1$.

For a holomorphic curve $f: \mathbf{C} \rightarrow \mathbf{P}^{n}$ and $k \in\{1, \ldots, n+1\}$, we define the $k$-proximity functions

$$
m_{k}(r, f)=\frac{1}{2 \pi} \int_{-\pi}^{\pi} \log \frac{1}{d_{k}\left(f\left(r e^{i t}\right)\right)} d t .
$$

So $m_{1} \geq m_{2} \geq \ldots \geq m_{n} \geq m_{n+1}=0$. Functions $m_{k}$ depend on $A$ which is not reflected in the notation. Proximity functions for flats of arbitrary codimension were considered for the first time by H. and J. Weyl's [15]. With this definition we have

Theorem 1. Let $f: \mathbf{C} \rightarrow \mathbf{P}^{n}$ be a linearly non-degenerate holomorphic curve. Let $A$ be an arbitrary finite complete set of hyperplanes. Then

$$
\sum_{k=1}^{n} m_{k}(r, f)+N_{1}(r, f) \leq(n+1) T(r, f)+S(r, f),
$$

where $S(r, f)$ is the same error term as in Cartan's theorem.

When $n=1$, we have

$$
m_{1}(r, f)=\frac{1}{2 \pi} \int_{-\pi}^{\pi} \max _{a \in A} \log \frac{1}{\operatorname{dist}\left(f\left(r e^{i t}\right), a\right)} d t+O(1),
$$

so in the case when $m(r, a, f)=o(T(r, f))$ for all but finitely many $a$, Theorem gives essentially the same as Yamanoi's result.

Let $f_{0}, \ldots, f_{n}$ be linearly independent polynomials whose maximal degree is $k$. Then there exist linear combinations $g_{0}, \ldots, g_{n}$ of these polynomials whose degrees satisfy $k_{0}<k_{1}<\ldots<k_{n}=k$. Then we have

$$
m_{j}(r, f)=\left(k-k_{j-1}\right) \log r+O(1), \quad T(r, f)=k \log r+O(1) .
$$

Computing the degree of the Wronskian $W\left(g_{0}, \ldots, g_{n}\right)$, we obtain

$$
N_{1}(r, f)=\left(\sum_{j=0}^{n} k_{j}-n(n+1) / 2\right) \log r+O(1) .
$$

Thus

$$
\sum_{j=1}^{n} m_{j}(r, f)+N_{1}(r, f)=(n+1) T(r, f)-\frac{n(n+1)}{2} \log r+O(1) .
$$

When $k$ is large, $T(r, f)$ is large in comparison with $\log r$, and we obtain a relation close to (13). So (13) can be considered as an extension of the formula for the degree of the Wronskian to the transcendental case, compare [14, Introduction, $\left.\left(\mathrm{II}^{\prime \prime}\right)\right]$.

The proof of Theorem 1 is a combination of Cartan's argument with the following elementary 
Lemma 1. Let $A$ be a finite complete set of hyperplanes in $\mathbf{P}^{n}$. Then there exists a constant $C>0$ depending only on $A$, such that for every $w \in \mathbf{P}^{n}$ we have

$$
\prod_{k=1}^{n} d_{k}(w) \geq C \min _{B} \prod_{a \in B} \operatorname{dist}(w, a)
$$

where the infimum is taken over all admissible systems $B=\left\{a_{1}, \ldots, a_{n+1}\right\}$ of hyperplanes in $A$.

Proof. First we notice that if $x \in F(A)$ and $\operatorname{codim} x=k$, then there exists an admissible subset $\left\{a_{1}, \ldots, a_{n+1}\right\} \in A$ such that $x=a_{1} \cap \ldots \cap a_{k}$. Indeed, by passing from hyperplanes to their defining vectors, this is equivalent to the familiar statement from linear algebra: if a finite set $A$ of vectors spans the space, then every linearly independent subset of $A$ can be completed to a basis consisting of vectors of $A$.

Now we prove the statement by contradiction. For $w$ not in the union of hyperplanes of $A$, we set

$$
\phi(w)=\frac{\prod_{k=1}^{n} d_{k}(w)}{\min _{B} \prod_{a \in B} \operatorname{dist}(w, a)} .
$$

Suppose that there is a sequence $w_{j}$ for which $\phi\left(w_{j}\right) \rightarrow 0$. By choosing a subsequence, we may assume that $w_{j} \rightarrow w_{\infty} \in \mathbf{P}^{n}$. If $w_{\infty}$ does not belong to any hyperplane $a \in A$, then $\phi\left(w_{\infty}\right)>0$, and we obtain a contradiction because $\phi$ is continuous in the complement of hyperplanes.

If $w_{\infty}$ belongs to some flat of $F(A)$, let $x \in F(A)$ be the flat of maximal codimension to which $w_{\infty}$ belongs. Then $d_{j}(w)$ are bounded away from zero for $w$ in a neighborhood $V$ of $w_{\infty}$ and $j>k=\operatorname{codim} x$. Suppose that $x=a_{1} \cap \ldots \cap a_{k}$. Then, by the remark in the beginning, there is an admissible system $B=\left\{a_{1}, \ldots, a_{n+1}\right\} \subset A$ beginning with $a_{1}, \ldots, a_{k}$, and $w_{\infty} \notin a_{j}$ for $j>k$ by definition of $x$. Then for $w \in V$, we have

$$
\prod_{j=1}^{n} d_{j}(w) \geq C_{1} \prod_{j=1}^{k} d_{j}(w) \geq C_{2} \prod_{j=1}^{k} \operatorname{dist}\left(w, a_{j}\right) \geq C_{3} \prod_{j=1}^{n+1} \operatorname{dist}\left(w, a_{j}\right) .
$$

This contradicts our assumption that $\phi\left(w_{j}\right) \rightarrow 0$ and proves the lemma.

Proof of Theorem 1. Fix a reduced representation of $f$. Normalize all hyperplane coordinates so that $\|\alpha\|=1$ in (1). Let

$$
u=\log \|f\|, \quad u_{a}=\log \left|g_{a}\right|, \quad a \in A .
$$

Then

$$
-\log \operatorname{dist}(f(z), a)=u(z)-u_{a}(z) .
$$

According to Lemma 1, for every $z \in \mathbf{C}$, we can find an admissible system $B(z),|B(z)|=$ $n+1$, in $A$ such that

$$
\begin{aligned}
-\sum_{k=1}^{n} \log \left|d_{k}(f(z))\right| & \leq-\sum_{a \in B(z)} \log \operatorname{dist}(f(z), a)+O(1) \\
& =(n+1) u(z)-\sum_{a \in B(z)} u_{a}(z)+O(1) .
\end{aligned}
$$


Let $W=W\left(f_{0}, \ldots, f_{n}\right)$ be the Wronskian determinant. If $B=\left\{a_{1}, \ldots, a_{n+1}\right\}$ is an admissible system, then

$$
\left|W_{B}\right|=\left|W\left(g_{a_{1}}, \ldots, g_{a_{n+1}}\right)\right|=C(B)|W| .
$$

Let

Then

$$
L_{B}(z)=\log ^{+}\left|\frac{W_{B}(z)}{\prod_{a \in B(z)}\left|g_{a}(z)\right|}\right| .
$$

$$
-\sum_{a \in B(z)} u_{a}(z) \leq-\log \left|W_{B}(z)\right|+\left|L_{B}(z)\right|+O(1) \leq-\log |W(z)|+R(z),
$$

where $R(z)$ is the sum of non-negative quantities $L_{B}(z)$ over all admissible systems of cardinality $n+1$. The Lemma on the Logarithmic derivative implies that

$$
\int_{-\pi}^{\pi} R\left(r e^{i t}\right) d t=S(r, f)
$$

see [4], [8, p. 222]. Jensen's formula gives

$$
\frac{1}{2 \pi} \int_{-\pi}^{\pi} \log \left|W\left(r e^{i t}\right)\right| d t=N_{1}(r, f)+O(1)
$$

and the definition of $T(r, f)$ can be rewritten as

$$
\frac{1}{2 \pi} \int_{-\pi}^{\pi} u\left(r e^{i t}\right) d t=T(r, f)+O(1)
$$

Combining (16) and (18), integrating over circles $|z|=r$, and using the last three equations we obtain

$$
\sum_{k=1}^{n} m_{k}(r, f)+N_{1}(r, f) \leq(n+1) T(r, f)+S(r, f) .
$$

This completes the proof of Theorem 1.

Now we compare Cartan's formulation of the SMT with Theorem 1.

Proposition. Let $A$ be a finite admissible system of hyperplanes, $|A| \geq n+1$, and $f$ a non-constant holomorphic curve whose image in not contained in any hyperplane of $A$. Then

$$
\sum_{a \in A} m(r, a, f) \leq \sum_{k=1}^{n} m_{k}(r, f)+O(1) .
$$

Proof. Let $A=\left\{a_{1}, \ldots, a_{q}\right\}$. Define $u$ and $u_{j}=\log \left|g_{a_{j}}\right|$ by formulas (15). Fix $z \in \mathbf{C}$ and order the functions $u_{j}$ by magnitude of $u_{j}(z)$,

$$
u_{j_{1}}(z) \leq u_{j_{2}}(z) \leq \ldots \leq u_{j_{q}}(z)
$$

where the $j_{k}$ depend on $z$. Then for $k \leq n$ we have

$$
u(z)-u_{j_{k}}(z)=-\log \operatorname{dist}\left(f(z), x_{k}\right)+O(1) \leq-\log d_{k}(f(z))+O(1),
$$

where $x_{k}=a_{j_{1}} \cap \ldots \cap a_{j_{k}}$, and the $O(1)$ depends only on $A$. For $k \geq n+1$ we obtain $u(z)-u_{j_{k}}(z)=O(1)$. Adding these inequalities we obtain

$$
\sum_{k=1}^{q} u(z)-u_{j_{k}}(z) \leq-\sum_{k=1}^{n} d_{k}(f(z))+O(1) .
$$


Integrating this inequality, over circles $|z|=r$ we obtain the statement of the proposition.

Remark. Unlike the usual proximity functions $m(r, a, f)$, the $m_{k}(r, f)$ can be substantially greater than $T(r, f)$. For example, if $f$ is the curve considered in the previous section, then $m_{1}(r, f)=2 T(r, f)+O(1)$. It is a challenging problem to obtain the exact upper estimates of the quantities

$$
\delta_{k}=\liminf _{r \rightarrow \infty} \frac{m_{k}(r, f)}{T(r, f)}
$$

for every $k \in[1, n]$. These are analogs of Nevanlinna defects. There is a conjecture that $\delta_{2} \leq 1$ for $n=2$.

\section{Curves defined by solutions of linear ODE}

Let $\mathfrak{F}$ be the set of all entire functions $y$ which satisfy differential equations of the form

$$
y^{(N)}+P_{N-1} y^{(N-1)}+\ldots+P_{0} y=0
$$

with polynomial coefficients $P_{j}$. This class contains exponential polynomials. For the curves of the form $f(z)=\left(e^{\lambda_{0} z}: \ldots: e^{\lambda_{n} z}\right)$ asymptotic equality holds in Cartan's SMT [3].

Theorem 2. Let $f: \mathbf{C} \rightarrow \mathbf{P}^{n}$ be a transcendental linearly non-degenerate holomorphic curve, whose homogeneous coordinates belong to $\mathfrak{F}$. Then there exists a finite complete system $A$ of hyperplanes such that

$$
\sum_{k=1}^{n} m_{k}(r, f)+N_{1}(r, f)=(n+1+o(1)) T(r, f), \quad r \rightarrow \infty .
$$

These curves are of finite order, so there is no exceptional set of $r$. The result seems to be new even for $n=1$.

To prove Theorem 2, we use the following two facts about the class $\mathfrak{F}$ :

1. $\mathfrak{F}$ is a differential ring [7]. This means that $\mathfrak{F}$ is closed under addition, multiplication and differentiation.

2. For every differential equation (19) and every $\theta$, there exists $\epsilon>0$, and $N$ linearly independent solutions $y_{1}, \ldots, y_{N}$ of $(19)$ such that

$$
y_{k}(z) \sim e^{Q_{k}\left(z^{1 / p}\right)} z^{s_{k} / p} \log ^{m_{k}} z, \quad z=r e^{i t}, \quad r \rightarrow \infty,
$$

uniformly with respect to $t$ when $|t-\theta| \leq \epsilon$. Here $Q_{k}$ are polynomials, $Q_{k}(0)=$ $0, p$ is a positive integer, and $s_{k}, m_{k}$ are integers. All triples $\left(Q_{k}, n_{k}, m_{k}\right), 1 \leq$ $k \leq N$, in (20) are distinct. For a proof we refer to [14].

We make some conclusions of this second fact:

a) Except for a finite set of rays, the asymptotics of $\left|y_{k}\right|$ are all distinct, in the sense that for $k \neq m$ the limit of $\left|y_{k} / y_{m}\right|$ as $|z| \rightarrow \infty$ is either zero or infinity on every ray, except finitely many rays.

b) For each $y \in \mathfrak{F}$ there exists a finite set $E \subset[0,2 \pi]$ such that for each $t \in$ $[0,2 \pi] \backslash E$ we have

$$
\left|y\left(r e^{i t}\right)\right| \sim c e^{Q\left(r^{1 / p}\right)} r^{s / p}(\log r)^{m}, \quad r \rightarrow \infty,
$$


where $c \in \mathbf{R} \backslash\{0\}, Q$ is a real polynomial, $p$ is a positive integer, and $s, m$ are integers.

In particular,

c) For every $t \in[0,2 \pi] \backslash E$, there exist $\rho=\rho(y, t)$ and $h=h(y, t) \neq 0$, such that there exists a limit

$$
\lim _{r \rightarrow \infty} \frac{\log \left|y\left(r e^{i t}\right)\right|}{r^{\rho}}=h(y, t) .
$$

This also holds for the elements of the ratio field $\mathfrak{R}$ of $\mathfrak{F}$.

The number $\rho(y, t)$ will be called the exponential order of $y$ on the ray $\left\{r e^{i t}: r>\right.$ $0\}$. If $y$ satisfies $(21)$ on this ray, then $\rho(y, t)=(\operatorname{deg} Q) / p$ and $h(y, t)$ is the coefficient of top degree of $Q$.

Fix a non-exceptional ray $L=\left\{r e^{i t_{0}}: r>0\right\}$. Then the solutions (20) can be ordered so that $y_{k}=o\left(y_{k+1}\right)$ as $|z| \rightarrow \infty$ on $L, 1 \leq k \leq N-1$. The ordering depends on the ray $L$, but the same ordering holds on nearby rays, so the ordering does not change in the sector between two adjacent exceptional rays.

Let $Y$ be the vector space of all solutions of equation (19). Then each element $u \in Y \backslash\{0\}$ satisfies $u \sim c y_{k}$ on $L$, with some $k$ and a constant $c \neq 0$. Moreover, if $u_{1} \sim c_{1} y_{k}$ and $u_{2} \sim c_{2} y_{k}$ then the linear combination $c_{2} u_{1}-c_{2} u_{2}=O\left(y_{k-1}\right)$.

Proof of Theorem 2. Let $f: \mathbf{C} \rightarrow \mathbf{P}^{n}$ be a linearly non-degenerate holomorphic curve whose homogeneous coordinates are functions of $\mathfrak{F}$. By [7, Satz. 1], we may assume that the coordinates satisfy one equation (19). Let $V \subset Y$ be the subspace spanned by the homogeneous coordinates. It consists of all functions $g_{a}=(a, f)$. Let us fix a non-exceptional ray $L$. Each element $w \in V \backslash\{0\}$ has an asymptotics $w \sim c y_{k}$ on $L$. Let $w_{1}, \ldots, w_{n+1}$ be the a maximal set of elements of $V$ with the property that $w_{j} \sim c_{j} y_{k_{j}}$ with $k_{j}$ pairwise distinct. We arrange the $w_{k}$ so that $w_{k}=o\left(w_{k+1}\right)$ as $|z| \rightarrow \infty z \in L$.

Consider the subspaces $V_{k} \subset V$ consisting of functions $w$ with the property $w=O\left(w_{k}\right)$ on $L$. We have

$$
V_{1} \subset V_{2} \subset \ldots \subset V_{n+1}=V
$$

and $\operatorname{dim} V_{k}=k$. So there exist hyperplanes $a_{1}, \ldots, a_{n+1}$ such that each $V_{k}$ is spanned by $g_{a_{1}}, \ldots, g_{a_{k}}$. These hyperplanes depend on the ray $L$, but they can be chosen the same for all rays in each sector complementary to the exceptional rays. Taking all these hyperplanes for all sectors we obtain an finite set $A$ of hyperplanes.

Now we compute the asymptotics of $\log d_{k}(f(z))$ on a fixed non-exceptional ray $L$. Let $a_{1}, \ldots, a_{n+1}$ be the hyperplanes associated to this ray in the previous paragraph. This set of hyperplanes is admissible, and we consider flats $x_{k}=a_{1} \cap \ldots \cap a_{k}$. Then

$$
\begin{aligned}
\log d_{k}(f(z)) & \leq \log \operatorname{dist}\left(f(z), x_{k}\right) \leq \max _{1 \leq j \leq k} \log \operatorname{dist}\left(f(z), a_{j}\right)+O(1) \\
& =\log \operatorname{dist}\left(f(z), a_{k}\right)+O(1)=u_{k}-u+O(1),
\end{aligned}
$$

where $u_{k}=\log \left|g_{a_{k}}\right|$ and $u=\log \|f\|$, as before. To obtain the estimate in the opposite direction, we argue by contradiction. Suppose that

$$
\log d_{k}\left(f\left(z_{j}\right)\right)-u_{k}\left(z_{j}\right)+u\left(z_{j}\right) \rightarrow-\infty
$$

for some sequence $z_{j} \in L, z_{j} \rightarrow \infty$. Then we can choose a subsequence, and a flat $x_{k} \in F(A), \operatorname{codim} x_{k}=k$, such that $d_{k}\left(f\left(z_{j}\right)\right)=\operatorname{dist}\left(f\left(z_{j}\right), x_{k}\right)$ for this subsequence 
$z_{j}$. Let $x_{k}=b_{1} \cap \ldots \cap b_{k}$. Then $b_{1}, \ldots, b_{k}$ is an admissible system, so the dimension of the span $S$ is $k$. On the other hand, for all $w \in S$, we will have $w=o\left(w_{k}\right)$, thus $S \subset V_{k-1}$, which is a contradicts $\operatorname{dim} V_{k-1}=k-1$.

We proved that for every non-exceptional ray

$$
-\sum_{k=1}^{n} \log d_{k}(f(z))=n u-\sum_{k=1}^{n} u_{k}+O(1) .
$$

Now we compute the asymptotics of the Wronskian $W=W\left(f_{0}, \ldots, f_{n}\right)$ on a nonexceptional ray. Using (17), it is sufficient to compute $W\left(g_{a_{1}}, \ldots, g_{a_{n+1}}\right)$. All these functions have asymptotics $g_{a_{k}} \sim w_{k}$.

Lemma 2. Let $w_{1}, \ldots, w_{m}$ be functions of $\mathfrak{F}$ with distinct asymptotics (21) on some non-exceptional ray. Then their Wronskian satisfies

$$
\log \left|W\left(w_{1}, \ldots, w_{m}\right)\right|=\sum_{j=1}^{m} \log \left|w_{j}\right|+O(\log r)
$$

on this ray.

Postponing the proof of this lemma, we finish the proof of Theorem 2. According to Lemma 2 we have

$$
\log |W(z)|=\sum_{j=1}^{n} u_{j}(z)+u(z)+O(\log |z|) .
$$

Combining (22) and (23) we obtain on our ray $L$

$$
\log |W(z)|-\sum_{j=1}^{n} \log d_{j}(f(z))=(n+1) u(z)+O(\log |z|) .
$$

Each term of this equation, except the error term is independent of $L$. The estimate of the error term is uniform with respect to $\arg z$, except at the small neighborhoods of the exceptional rays. Integrating over circles $|z|=r$ we obtain the statement of our theorems, because the contribution of the small neighborhoods of finitely many exceptional rays is $o(T(r, f))$.

Proof of Lemma 2. Evidently $W=W\left(w_{1}, \ldots, w_{m}\right) \in \mathfrak{F}$. We use the notation

$$
\mathcal{L}\left(w_{1}, \ldots, w_{m}\right)=\frac{W\left(w_{1}, \ldots, w_{m}\right)}{w_{1} \ldots w_{m}} .
$$

In view of $(21)$ it is sufficient to prove that $\rho\left(\mathcal{L}\left(w_{1} \ldots w_{m}\right)\right)=0$.

As $\mathcal{L}$ is a determinant consisting of logarithmic derivatives of functions of the class $\mathfrak{F}$, we always have $\rho(\mathcal{L}) \leq 0$ by the lemma on the logarithmic derivative [8]. It remains to prove that $\rho(\mathcal{L}) \geq 0$.

We prove this by induction in $m$. The statement is evident when $m=1$. Suppose that it holds for all Wronskians of size at most $m-1$. Assume by contradiction that $\rho\left(\mathcal{L}\left(w_{1}, \ldots, w_{m}\right)\right)<0$, and order our functions $w_{j}$ so that $w_{j}=o\left(w_{j+1}\right)$, on the ray $L$. Define functions $A_{j}$ as solutions of the following system of linear equations

$$
\sum_{j=1}^{m-1} A_{j} w_{j}^{(k)}=w_{m}^{(k)}, \quad k=0, \ldots, m-2 .
$$


By Cramer's rule,

$$
A_{j}= \pm \frac{W_{j}}{W_{m}}
$$

where $W_{j}$ is the Wronskian of size $m-1$ made of functions $w_{i}$ with $i \neq j$. We use the formula for differentiation of the logarithm of the quotient of Wronskians [12, Part VII, Probl. 59], [8, p. 251]

$$
\frac{d}{d z} \log \left(\frac{W_{j}}{W_{m}}\right)=\frac{W_{j, m} W}{W_{j} W_{m}}=\frac{\mathcal{L}_{j, m} \mathcal{L}}{\mathcal{L}_{j} \mathcal{L}_{m}},
$$

where $W_{j, m}$ is the Wronskian of size $m-2$ with $w_{j}$ and $w_{m}$ deleted, and $W$ is our Wronskian of size $m$. Notation $\mathcal{L}, \mathcal{L}_{j}, \mathcal{L}_{j, m}$ has similar meaning. Using the induction assumption, we conclude that the right hand side of (24) has negative exponential order. Integrating with respect to $z$ along the ray $L$ we obtain $A_{j} \sim c_{j}, 1 \leq j \leq m-1$, where $c_{j} \neq 0$ are constants. So we have

$$
w_{m}=\sum_{j=1}^{m-1}\left(c_{j}+o(1)\right) w_{j} .
$$

This contradicts our assumption that $w_{j}=o\left(w_{m}\right)$. This contradiction completes the proof of the lemma.

Remark. A special case of Theorem 2 is that the homogeneous coordinates of $f$ are linearly independent solutions of $(7)$ with $N=n+1$. In this case we have $N_{1}(r, f)=0$. For such curves Theorem 2 gives

$$
\sum_{k=1}^{n} m_{k}(r, f)=(n+1+o(1)) T(r, f) .
$$

These curves are analogous to meromorphic functions considered in $[9,10]$.

The author thanks the referee whose remarks helped to improve the exposition.

\section{References}

[1] Abramowitz, M., and I. Stegun: Handbook of mathematical functions with formulas, graphs, and mathematical tables. - U.S. Government Printing Office, Washington D.C., 1964.

[2] Ahlfors, L.: Zur Theorie der Uberlagerungsflächen. - Acta Math 65:1, 1935, 157-194.

[3] Ahlfors, L.: The theory of meromorphic curves. - Acta Soc. Sci. Fenn. 3-4, 1941, 1-31.

[4] Cartan, H.: Sur les zéros des combinaisons linéaires de $p$ fonctions holomorphes données. Mathematica, Cluj, 7, 1933, 5-31.

[5] Eremenko, A.: Extremal holomorphic curves for defect relations. - J. Anal. Math. 74, 1998, $307-323$.

[6] Eremenko, A.: A Toda lattice in dimension 2 and Nevanlinna theory. - J. Math. Phys. Anal. Geom. 31, 2007, 39-46.

[7] Frank, G., and H. Wiтtich: Zur Theorie linearer Differentialgleichungen im Komplexen. Math. Z. 130, 1973, 363-370.

[8] LANG, S.: Introduction to complex hyperbolic spaces. - Springer, NY, 1987.

[9] Nevanlinna, F.: Über eine Klasse meromorpher Funktionen. - Comptes Rendus de Septiéme Congr̀ess Math. Scand., Oslo 1929, A. W. Brøggers Boktrykkeri, Oslo, 1930. 
[10] Nevanlinna, R.: Über Riemannsche Flächen mit endlich vielen Windungspunkten. - Acta Math. 58, 1932, 295-373.

[11] Petrenko, V.: Entire curves. - Vyshcha shkola, Kharkiv, 1984 (in Russian).

[12] Pólya, G., and G. Szegö: Aufgaben und Lehrsätze aus der Analysis, Band II. - Julius Springer, Berlin, 1925.

[13] Teichmüller, O.: Eine Umkehrung des zweiten Hauptsatzes der Wertverteilungslehre. Deutsche Math. 2, 1937, 96-107.

[14] Wasow, W.: Asymptotic expansions for ordinary differential equations. - Interscience Publ., NY, 1965.

[15] Weyl, H., and J. Weyl: Meromorphic curves. - Ann. of Math. 39:3, 1938, 516-538.

[16] Wiтtich, H.: Neuere Untersuchungen über eindeutige analytische Funktionen. - Springer, Berlin, 1955.

[17] Yamanoi, K.: Zeros of higher derivatives of meromorphic functions in the complex plane. Proc. Lond. Math. Soc. (3) 106:4, 2013, 703-780.

[18] Yamanoi, K.: On a reversal of the Second Main Theorem for meromorphic functions of finite order. - In: Proceedings of the 19th ICFIDCAA (Hiroshima, 2011), edited by K. Matsuzaki and T. Sugawa, Tohoku Univ. Press, 2012, 75-83.

Received 27 October 2013 • Accepted 21 February 2014 